\title{
Óptimo aprovechamiento de las sesiones de laboratorio
}

\author{
Jennifer Gonzalez Ausejo ${ }^{a}$, Raquel Oliver Valls ${ }^{a}$, José Gámez Pérez ${ }^{a}$ y Luís Cabedo \\ Mas $^{a}$
} a Departamento de Sistemas Industriales y Diseño, Universitat Jaume I, ausejo@uji.es, roliver@uji.es,
gamez@uji.es y lcabedo@uji.es.

\begin{abstract}
This paper shows the teaching experience applied to the laboratory sessions of the subject of Science and Technology of Materials that is taught in the second year of the degrees of Electrical Engineering, Mechanical Engineering and Industrial Technologies Engineering in the Universitat Jaume I. The purpose of this experience is to facilitate the learning of the basic theoretical knowledge related to laboratory practice to develop and to make the students aware of the importance of preparing the practice prior to its development. This has been done by developing and using means or transmission formats of the contents to know before the practice which are more attractive and visual than the existing practice script.
\end{abstract}

Keywords: previous knowledge, visual, evaluation, methodology

\begin{abstract}
Resumen
En este trabajo se muestra la experiencia docente aplicada a las sesiones de laboratorio de la asignatura de Ciencia y Tecnología de Materiales que se imparte en segundo curso de los grados de Ingeniería Eléctrica, Mecánica y en Tecnologías Industriales en la Universitat Jaume I. El propósito de esta experiencia es facilitar el aprendizaje de los conocimientos teóricos básicos relacionados con la práctica de laboratorio a desarrollar y sensibilizar a los alumnos sobre la importancia de preparar la práctica previamente a su desarrollo. Esto se ha realizado mediante el desarrollo y uso de medios o formatos de trasmisión de los contenidos a conocer previamente a la práctica que sean más atractivos y visuales que el guion de prácticas existente actualmente.
\end{abstract}

Palabras clave: conocimientos previos, visual, evaluación, metodología

\section{Introducción}

El planteamiento de clases prácticas y de laboratorio en cualquier asignatura permite reforzar los conocimientos expuestos en las clases teóricas relacionando los conceptos teóricos con la experiencia. Por lo que si las sesiones de laboratorio o prácticas se aprovechan adecuadamente son una forma rápida y eficiente de aprender. Sin embargo, el aprovechamiento de las practicas se puede ver limitado por el desconocimiento de los fundamentos teóricos implicados en la misma. 
La gran evolución e implementación de las tecnologías informáticas de la comunicación (TIC) en los últimos años ha permitido avanzar y modificar las metodologías docentes, adaptándolas a la evolución de los sistemas de comunicación actuales más llamativos y atractivos para las nuevas generaciones (Salinas, 2004). Esta nueva generación de alumnos utiliza con total soltura las nuevas tecnologías como herramienta útil y eficiente de estudio.

Debido a este contexto, las metodologías docentes deben ser adaptadas a estos nuevos escenarios de aprendizaje utilizando las TIC como herramienta docente para la trasmisión de los conocimientos, a la vez que se capta su atención en mayor medida que lo que lo hace un simple texto.

\section{Objetivos}

El objetivo principal de este trabajo es facilitar a los alumnos el aprendizaje de los conceptos teóricos que se van a aplicar durante el desarrollo de las prácticas de laboratorio de la asignatura de Ciencia y Tecnología de Materiales por medio de la modificación de la metodología docente utilizada, para el óptimo aprovechamiento de la práctica.

Concretamente los objetivos planteados con esta metodología son:

- Facilitar el aprendizaje de los conocimientos teóricos básicos relacionados con la práctica de laboratorio a desarrollar.

- Evaluar los conocimientos previos de los alumnos antes de la manipulación de los equipos en el laboratorio.

- Concienciar a los alumnos de la importancia de los contenidos teóricos previos con anterioridad al desarrollo de la práctica.

\section{Desarrollo de la innovación}

La asignatura de Ciencia y Tecnología de Materiales, que se imparte en segundo curso de los grados de Ingeniería Eléctrica, Mecánica y en Tecnologías Industriales en la Universitat Jaume I, es una asignatura troncal que consta 4 créditos de enseñanza teórica y 1 crédito de enseñanza práctica.

Las enseñanzas prácticas consisten en clases de laboratorio donde los alumnos utilizan los equipos y técnicas de evaluación de las propiedades de los materiales para reforzar y/o complementar los contenidos expuestos en las clases teóricas.

Para el óptimo aprovechamiento de las sesiones prácticas se requiere del conocimiento de los fundamentos teóricos concretos implicados en la práctica, el funcionamiento y el fundamento de los equipos e instrumentos de medida, etc. a utilizar durante ésta. Estos contenidos mínimos han sido redactados en un guion de prácticas.

Sin embargo, actualmente el alumnado no prepara este material con anterioridad a la asistencia al aula. Ello ha conducido a que el profesor/a realice una introducción teórica como una clase magistral en el laboratorio con el fin que el alumnado conozca los

(c)) BY-NC-ND 2017, Universitat Politècnica de València 
fundamentos teóricos mínimos necesarios para el aprovechamiento de la práctica (resumen de la metodología actual mostrada en la Tabla 1). Los resultados de la aplicación de esta metodología durante diversos cursos muestran que no hay un óptimo aprovechamiento de las sesiones de prácticas.

Tabla. 1: Comparativa de la metodología docente aplicada en cursos anteriores y la metodología propuesta

\begin{tabular}{|c|c|c|c|}
\hline & \multicolumn{2}{|c|}{ Actualmente } & \multirow{2}{*}{ Propuesta } \\
\hline & Ideal & Realidad & \\
\hline $\begin{array}{l}\text { Material: conceptos } \\
\text { teóricos que se van a } \\
\text { aplicar durante el } \\
\text { desarrollo de las } \\
\text { prácticas }\end{array}$ & \multicolumn{2}{|c|}{ Guion de prácticas: texto } & $\begin{array}{c}\text { Video/audio/ } \\
\text { presentación power } \\
\text { point }\end{array}$ \\
\hline $\begin{array}{c}\text { En casa: previamente } \\
\text { a la práctica }\end{array}$ & $\begin{array}{l}\text { Estudio del guion } \\
\text { de prácticas }\end{array}$ & - & $\begin{array}{c}\text { Estudio del nuevo } \\
\text { material } \\
\text { desarrollado }\end{array}$ \\
\hline \multirow{2}{*}{$\begin{array}{l}\text { En el aula/ } \\
\text { laboratorio }\end{array}$} & Puesta en común & Clase magistral & $\begin{array}{c}\text { Tests de } \\
\text { autoevaluación }\end{array}$ \\
\hline & \multicolumn{3}{|c|}{ Desarrollo práctica } \\
\hline $\begin{array}{c}\text { En casa: } \\
\text { posteriormente a la } \\
\text { práctica }\end{array}$ & \multicolumn{3}{|c|}{ Redacción memoria de práctica } \\
\hline
\end{tabular}

Tabla. 2: Propuesta de actuación y objetivos de la metodología planteada.

\section{\begin{tabular}{l|l} 
Propuesta de actuación & Objetivo
\end{tabular}}

Preparación del material nuevo $\quad$ Facilitar el aprendizaje de los conocimientos teóricos desarrollado: video/audio/ presentación power point. básicos relacionados con la práctica de laboratorio a desarrollar.

Evaluar los conocimientos previos de los alumnos antes de la manipulación de los equipos en el laboratorio.

Tests de autoevaluación Concienciar a los alumnos de la importancia de los contenidos teóricos previos con anterioridad al desarrollo de la práctica.

Por ello, en este trabajo se plantea la modificación de la metodología docente utilizada para un óptimo aprovechamiento de la práctica.

Para lograr los objetivos previamente expuestos, en este proyecto se han realizado distintos cambios en la metodología (ver Tabla 1 y 2):

- Por un lado, se han desarrollado materiales docentes utilizando distintos formatos o medios de difusión para su estudios previamente a la práctica en sustitución del guion de prácticas y la explicación magistral al inicio de la sesión de prácticas.

- Además se han realizado tests de autoevaluación en el aula para garantizar que se lleva a cabo la preparación previa de la práctica y evaluar el grado de 
conocimiento de los contenidos mínimos para la manipulación de los equipos de laboratorio.

Concretamente los contenidos teóricos, procedimientos a desarrollar durante la práctica, normas de laboratorio, etc., han sido expuestos por medio de audios, presentaciones de power point y vídeos.

Para cada práctica se ha desarrollado el material con un formato distinto, adaptándose a éste en función del contenido concreto de la práctica a desarrollar. El fin último es determinar si los tres formatos propuestos son útiles y qué ventajas e inconvenientes plantean para que los alumnos, con estos materiales, puedan revisar y conocer de manera más sencilla y atractiva los contenidos de la sesión de prácticas.

Los materiales desarrollados están disponibles de manera permanente en la plataforma Moodle (Aula Virtual), de modo que los alumnos pueden consultarlos tantas veces como lo crean conveniente para afianzar los conocimientos y elaborar la memoria de prácticas con mayor grado de aprendizaje real o efectivo.

Los resultados de los tests son tenidos en cuenta en la nota final del laboratorio.

\section{Resultados}

Para evaluar el impacto de la propuesta impulsada en este proyecto sobre el aprovechamiento de las sesiones de prácticas por parte de los alumnos, se ha realizado una amplia encuesta al finalizar la asignatura.

Se ha tenido en cuenta la visión crítica de los alumnos a través de sus opiniones para analizar la propuesta y así poder mejorar la efectividad de la práctica de laboratorio mediante este tipo de material en cursos sucesivos.

Adicionalmente, se ha realizado un breve estudio estadístico de las calificaciones de los alumnos en cursos pasados y en el presente curso, habiendo sido aplicada la metodología expuesta en este proyecto.

\subsection{Implicación de los alumnos}

Los alumnos desde el primer momento han mostrado interés por la metodología desarrollada, no han puesto objeción en la realización de los test de evaluación de los conocimientos previos a la realización de la práctica.

Los resultados de los test muestran que los alumnos han preparado concienzudamente la práctica ya que el $89 \%$ de los alumnos ha realizado al menos el $75 \%$ del cuestionario bien. Además, la nota media de las memorias de prácticas se ha incrementado, con respecto a otros grupos de años anteriores, realizando las mismas prácticas.

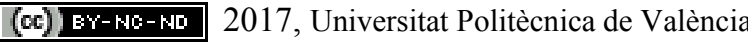




\subsection{Valoración de las encuestas}

La Tabla 3 muestra la encuesta de satisfacción del estudiantado sobre la metodología aplicada. Todas las cuestiones se han valorado en una escala Likert 5, donde 1 es "no adecuado o totalmente en desacuerdo" y 5 "adecuado o totalmente de acuerdo". Además, se han realizado cuestiones de respuesta si/no para evaluar el cambio en la concepción de la importancia de la preparación de la práctica previamente a su desarrollo. También se ha preguntado a los alumnos sobre si esta metodología debería implementarse en otras asignaturas y/o cursos sucesivos. Con el fin de conocer más información se han realizado preguntas abiertas en el cuestionario. Los resultados de las encuestas (valoración media/ contestación si/no) se muestran junto a las cuestiones en la Tabla 3.

El orden de las cuestiones ha sido definido meticulosamente para favorecer la reflexión del alumno durante el desarrollo del mismo.

Todas las cuestiones obtuvieron puntuaciones superiores al 4 como puede ser observado en la Tabla 3.

Tabla. 3: Cuestionario de valoración de la metodología aplicada

\begin{tabular}{|c|c|}
\hline Cuestión & $\begin{array}{l}\text { Valoración media } \\
\quad(\text { de } 1 \text { a } 5) \\
\end{array}$ \\
\hline $\begin{array}{l}\text { 1. Valora si los materiales utilizados para exponer el contenido } \\
\text { teórico de la práctica } X \text { te han parecido adecuados. }\end{array}$ & $\begin{array}{c}\text { P1: 4,44 (audio) } \\
\text { P2: 4,31(video) } \\
\text { P3: 4,06 (power } \\
\text { point) } \\
\text { P4: 4,19 (vídeo) }\end{array}$ \\
\hline $\begin{array}{l}\text { 2. Los materiales utilizados para la exposición del contenido teórico } \\
\text { de la práctica tienen una duración adecuada }\end{array}$ & 4,06 \\
\hline $\begin{array}{l}\text { 3. El formato de los materiales proporcionados han hecho más } \\
\text { ameno el estudio de la práctica con respecto a una guía escrita de } \\
\text { la memoria }\end{array}$ & 4,44 \\
\hline $\begin{array}{l}\text { 4. Conocer los contenidos teóricos básicos, objetivos, procedimiento } \\
\text { a desarrollar en las prácticas, etc., previamente al desarrollo de la } \\
\text { práctica favorece el entendimiento de las prácticas }\end{array}$ & 4,81 \\
\hline $\begin{array}{l}\text { 5. La realización del test previamente al desarrollo de la práctica } \\
\text { permite hacer una autoevaluación de los conocimientos } \\
\text { adquiridos. }\end{array}$ & 4,31 \\
\hline $\begin{array}{l}\text { 6. La realización del test y su resolución al inicio de la sesión de } \\
\text { prácticas me ha permitido completar el conocimiento necesario } \\
\text { para la correcta comprensión de la práctica. }\end{array}$ & 4,38 \\
\hline $\begin{array}{l}\text { 7. Conocer y evaluar los conceptos tratados en la práctica } \\
\text { previamente al desarrollo de la memoria ha favorecido unos } \\
\text { mejores resultados finales en la memoria de prácticas (nota de la } \\
\text { memoria). }\end{array}$ & 4,50 \\
\hline $\begin{array}{l}\text { 8. Disponer del material explicativo de la práctica ha facilitado la } \\
\text { redacción de la memoria de prácticas. }\end{array}$ & 4,75 \\
\hline $\begin{array}{l}\text { 9. Antes de realizar esta asignatura, ¿creías necesario conocer el } \\
\text { contenido teórico básico, objetivos, procedimiento a desarrollar } \\
\text { en las prácticas, etc., antes de iniciar la práctica? }\end{array}$ & $\begin{array}{l}\text { Si: } 50 \% \\
\text { No: } 50 \%\end{array}$ \\
\hline
\end{tabular}




\begin{tabular}{|c|c|}
\hline Cuestión & $\begin{array}{l}\text { Valoración media } \\
\text { (de } 1 \text { a } 5 \text { ) }\end{array}$ \\
\hline $\begin{array}{l}\text { 10. Contestación No en la cuestión anterior: } \\
\text { ¿Tras realizar las prácticas aplicando esta metodología, has } \\
\text { cambiado de opinión? }\end{array}$ & $\begin{array}{c}\text { Si: } 87,5 \% \\
\text { No: } 12,5 \%\end{array}$ \\
\hline $\begin{array}{l}\text { 11. Contestación Si en la cuestión anterior: } \\
\text { ¿Ha servido la metodología utilizada en clase para que creas } \\
\text { aún más necesario conocer el contenido teórico básico, } \\
\text { objetivos procedimiento a desarrollar en las prácticas, etc., antes } \\
\text { de iniciar la práctica? }\end{array}$ & $\begin{array}{l}\text { Si: } 87,5 \% \\
\text { No: } 12,5 \%\end{array}$ \\
\hline $\begin{array}{l}\text { 12. Valora el esfuerzo extra que te ha supuesto preparar la práctica } \\
\text { antes de la sesión. }\end{array}$ & 3,13 \\
\hline $\begin{array}{l}\text { 13. En general, valora el grado de satisfacción con la metodología } \\
\text { aplicada. }\end{array}$ & 4,56 \\
\hline $\begin{array}{l}\text { 14. ¿Te gustaría que se aplicase esta metodología en las prácticas de } \\
\text { otras asignaturas? }\end{array}$ & $\begin{array}{l}\text { Si: } 87,5 \% \\
\text { No: } 12,5 \%\end{array}$ \\
\hline \multicolumn{2}{|l|}{ Preguntas abiertas } \\
\hline \multicolumn{2}{|c|}{$\begin{array}{l}\text { En caso que los materiales no te pareciesen adecuados o no te ayudasen al estudio puedes } \\
\text { dar las razones o exponer alternativas que te parezcan más idóneas. }\end{array}$} \\
\hline \multicolumn{2}{|c|}{$\begin{array}{c}\text { Finalmente, ¿qué ventajas consideras que ha supuesto esta metodología en tu proceso de } \\
\text { aprendizaje? }\end{array}$} \\
\hline \multicolumn{2}{|c|}{ Señala aspectos qué cambiarías de la metodología aplicada. } \\
\hline
\end{tabular}

De acuerdo con las valoraciones de los alumnos todos los formatos utilizados para la trasmisión de los contenidos de la práctica son adecuados y han sido valorados positivamente (cuestión 1). A partir de los comentarios de los alumnos (preguntas abiertas) se extrae que los vídeos son los medios de exposición de los contenidos teóricos más adecuados para el contenido específico de esta asignatura: "los materiales que incluían vídeo los recordaba más fácilmente, además de ser más ameno de estudiar”. Sin embargo, los audios son valorados por los alumnos como un material de estudio que requiere de una mayor concentración para la comprensión de los contenidos expuestos, además que supone una mayor dificultad el hecho de mantener la concentración durante toda la duración del audio en ausencia de imagen.

En cuanto a la duración de los audios/ vídeos (cuestión 2), la utilización de entre 10 y 13 min se ha valorado positivamente.

Otras conclusiones extraídas de la encuesta son que el formato de los materiales desarrollados (cuestión 3), audios, videos y presentaciones power point, captan mayor atención que un simple texto (guion de prácticas convencional) lo que hace más ameno el estudio de la práctica.

Los alumnos han tomado consciencia de la necesidad y utilidad de conocer los contenidos teóricos básicos, objetivos, procedimiento a desarrollar en las prácticas, etc., con anterioridad al desarrollo de la práctica, como se deriva de la alta puntuación de la cuestión 4 y de las preguntas directas 9 y 10 o 11 . De estas contestaciones se deduce que, antes de la experiencia desarrollada, el $50 \%$ de los alumnos no creían necesario conocer el contenido teórico básico, objetivos, procedimiento a desarrollar en las prácticas, etc., con antelación a 
la práctica. Sin embargo, el $87.5 \%$ de estos alumnos han cambiado su opinión tras la experiencia docente realizada. Además, aquellos alumnos que antes de la experiencia creían necesaria la preparación previa de la práctica, tras ésta el $87.5 \%$ ha reforzado su posición. De los comentarios de los alumnos, se extrae que el desarrollo de la asignatura, trabajando los contenidos teóricos previos al desarrollo de la práctica, favorece el entendimiento de las mismas.

En cuanto a la evaluación por medio de un test de los conocimientos adquiridos previamente al desarrollo de la práctica mediante los materiales desarrollados (cuestiones 5 a 8), los alumnos han valorado positivamente esta estrategia como método de autoevaluación. Ya que mediante el test han podido comprobar qué conocimientos de los expuestos en el material de estudio han sido adquiridos y cuáles requerían de un mayor refuerzo. Esto les ha permitido la correcta comprensión de la práctica. Asimismo, la realización de la evaluación previa al desarrollo de la práctica ha favorecido unos mejores resultados finales en la memoria final, ya que los errores conceptuales evidenciados en cursos anteriores no han sido observados en este caso.

Los alumnos también han expuesto que el hecho de disponer de los materiales explicativos de la práctica para la redacción de la memoria ha facilitado la tarea de redacción, análisis y discusión de los resultados experimentales.

Para finalizar la encuesta, se han realizado unas preguntas de valoración global de la metodología aplicada (cuestiones 12 a 14), obteniéndose que el grado de satisfacción global es del 4.56 (cuestión 13).

Se les ha instado a que aportasen puntos de mejora y ventajas que supone esta metodología. Estos son algunos de los comentarios sobre las ventajas / valoraciones de la experiencia que aporta la metodología utilizada frente a la metodología anterior:

- Iba muy preparado a las prácticas, sabiendo lo que iba a hacer y qué iba a pasar. Ayudaba mucho a realizar la memoria. En lo que más lo he notado es en saber qué estaba haciendo y no estar paseándome por el laboratorio sin más.

- Llevar los conceptos claros al laboratorio y aclarar detalles solamente durante la sesión.

- Me ha permitido obtener más conocimientos durante el laboratorio, mejorando así mi comprensión global de la asignatura.

- La facilidad de entender los procesos que se van a llevar a cabo y comprender mejor la práctica en general.

- Considero que he aprendido mucho más con esta metodología que con lo que normalmente hacemos en cualquier otra clase de laboratorio (guion de la práctica y breve explicación del profesor). De esta manera sabemos ya antes de empezar la sesión el procedimiento general que vamos a seguir, así como lo que se pretende con ello, de forma que el tiempo de laboratorio se optimiza y el/la profesor/a puede remarcar aquello que es de más importancia en lugar de tener que explicar todo de forma general.

- Óptimo aprovechamiento de las sesiones de laboratorio. 
En cuanto a las sugerencias planteadas por los alumnos para la mejora de la experiencia en cursos sucesivos, los estudiantes han comentado:

- Intentaría hacer más simplificados los PowerPoints, no mucho más porque sino no quedarían claros los conceptos. Pero en ocasiones se hacía muy pesado, sobretodo el escuchar el audio.

- No cambiaría nada (repetida varias veces).

Por último, se ha preguntado si les gustaría que se aplicase esta metodología en las prácticas de otras asignaturas (cuestión 14), obteniéndose que el 87.5\% de los alumnos apoyan esta iniciativa, aún suponiéndoles un esfuerzo extra la preparación de la práctica, valorado con un 3,13 sobre 5 (cuestión 12).

\subsection{Estudio estadístico de las calificaciones de los alumnos}

Con el fin de aportar datos cuantitativos que respalden la idoneidad de la metodología de trabajo expuesta en las sesiones prácticas de laboratorio, se ha desarrollado un breve estudio estadístico de las calificaciones de los alumnos en los últimos cuatro cursos escolares. Los datos estadísticos se muestran en la Tabla 4.
Tabla. 4: Comparativa de los valores de media, mediana y desviación estándar de las calificaciones de los últimos 4 cursos escolares de los alumnos de asignatura de Ciencia y Tecnología de Materiales de los grados de Ingeniería Eléctrica, Mecánica y en Tecnologías Industriales en la Universitat Jaume I.

\begin{tabular}{|c|c|c|c|c|}
\hline $\begin{array}{c}\text { Curso } \\
\text { académico }\end{array}$ & $2013 / 2014$ & $2014 / 2015$ & $2015 / 2016$ & $2016 / 2017$ \\
\hline Media & 7,7 & 8,3 & 7,3 & 8,7 \\
\hline Mediana & 8,1 & 8,1 & 8,1 & 8,5 \\
\hline $\begin{array}{c}\text { Desviación } \\
\text { estandar }\end{array}$ & 1,4 & 0,3 & 0,6 & 0,7 \\
\hline
\end{tabular}

Los resultados estadísticos mostrados en la Tabla 4 muestran una clara mejora de las calificaciones de los alumnos al ser aplicada la metodología propuesta para las sesiones prácticas de laboratorio con respecto a los cursos anteriores. Los valores más elevados de media y mediana de las calificaciones del curso 2016/2017 con respecto a los obtenidos para los tres cursos anteriores, en los que se aplicaba la metodología expuesta en la Tabla 1 (Realidad) demuestran la mejora del proceso de aprendizaje y rendimiento de los alumnos con respecto a la metodología de trabajo aplicada en cursos anteriores.

\section{Conclusiones}

Del presente trabajo se puede concluir que el aprendizaje de los conocimientos previos de una práctica de laboratorio mediante materiales docentes basados en audios, vídeos y presentaciones interactivas, y su evaluación con anterioridad al desarrollo de la práctica de laboratorio, favorece el aprendizaje de los conocimientos teóricos básicos relacionados con la práctica de laboratorio. 
Adicionalmente esta metodología ha resultado en la concienciación de los estudiantes en la importancia de la preparación de la práctica previamente al desarrollo de la misma.

Se ha constatado que la metodología aplicada supone el aprovechamiento óptimo de las sesiones de laboratorio, dando lugar a la mayor comprensión global de la asignatura de materiales, como puede ser observado por el incremento de las calificaciones medias y la mediana tras ser aplicada la metodología expuesta.

\section{Referencias}

SALINAS, J. (2004). Innovación docente y uso de las TIC en la enseñanza universitaria. Revista de Universidad y Sociedad del Conocimiento (RUSC). [artículo en línea]. UOC. Vol. 1, no 1. [Fecha de consulta: 31/03/17]. <http://www.uoc.edu/rusc/dt/esp/ salinas1104.pdf> 